\title{
Outcomes of active surveillance for clinically localized prostate cancer in a middle eastern tertiary care center
}

\author{
Mohammad Hout ${ }^{1}$, Ali Merhe ${ }^{1}$, Nassib Abou Heidar ${ }^{1}$, Jose M El-Asmar ${ }^{1}$, Wassim Wazzan ${ }^{1}$, \\ Bassel Bachir ${ }^{1}$, Rola Jaafar ${ }^{2}$, Albert El Hajj ${ }^{1}$, Muhammad Bulbul ${ }^{1}$ \\ ${ }^{1}$ Department of Surgery, Division of Urology, American University of Beirut Medical Center, Beirut, Lebanon; \\ ${ }^{2}$ Department of Surgery, American University of Beirut Medical Center, Beirut, Lebanon.
}

\begin{abstract}
Summary Background: The aim of our study was to evaluate the outcome of active surveillance
\end{abstract} (AS) for prostate cancer for a cohort of patients at our institution.

Methods: A total of 43 patients with low risk prostate cancer were enrolled in an active surveillance pilot program at our institution between 2008 and 2018. Follow up protocols included: periodic prostate specific antigen (PSA), digital rectal examination (DRE), multiparametric MRI, and prostate biopsy at one year. Pertinent parameters were collected, and descriptive statistics were reported along with a subset analysis of patients that dropped out of the protocol to receive active treatment for disease progression.

Results: Out of 43 eligible patients, $46.5 \%$ had a significant rise in follow up PSA. DRE was initially suspicious in $27.9 \%$ of patients, and none had any change in DRE on follow up. Initially, prostate MRIs showed PIRADS 3, 4, and 5 in 14\%, $37.2 \%$, and $11.6 \%$ respectively, while $23.2 \%$ had a negative initial MRI. 14\% did not have an MRI. Upon follow up, $18.6 \%$ of patients had progression on MRI. Initial biopsies revealed that $86 \%$ were classified as WHO group 1, while $14 \%$ as WHO group 2. With regards to the follow up biopsies, $11.6 \%$ were upgraded. $20.9 \%$ of our patients had active treatment; $44.4 \%$ due to upgraded biopsy results, $22.2 \%$ due to PSA progression, $22.2 \%$ due to strong patient preference, and $11.1 \%$ due to radiologic progression.

Conclusions: For selected men with low risk prostate cancer, AS is a reasonable alternative. The decision for active treatment should be tailored upon changes in PSA, DRE, MRI, and biopsy results.

KEY WORDS: Active surveillance; Prostate cancer; Men's health; Screening; Prostate specific antigen.

Submitted 14 May 2021; Accepted 19 July 2021

\section{INTRODUCTION}

Active surveillance (AS) as a management strategy for low grade prostate cancer is a relatively new approach increasingly utilized by clinicians in the light of better comprehension of low-grade prostate cancer behavior. It is a dynamic surveillance strategy that may shift into a direct curative intervention (1). The main goal is to follow-up patients with clinical parameters such as prostate specific antigen (PSA), digital rectal examination (DRE), imaging and biopsy according to predetermined protocols. The impor- tance of AS lies in evading unnecessary treatments, and so their potentially detrimental side effects, through careful surveillance at specific intervals to disease progression and need for intervention (1). The rationale justified by the slow and indolent course of low-grade prostate cancer $(2,3)$. Moreover, observational studies revealed no significant advantage in patients that underwent surgery versus active surveillance $(4,5)$. In particular, the PROTECT trial showed a similar 10-year Prostate cancer specific survival in patients with localized low-grade prostate cancer that underwent monitoring, surgery, or radiation therapy (6). Our purpose is to evaluate our active surveillance protocol and its outcomes in our Middle Eastern cohort over a 10year span. To our knowledge, this is the first AS outcome data reported outside of North America and Europe.

\section{Methods}

After obtaining institutional review board approval, we performed a retrospective review of our prostate cancer patients' data at the American University of Beirut- Medical Center (AUBMC), a Middle Eastern tertiary care center, over a span of 10 years (2008-2018). 43 of them satisfied the inclusion criteria into our institution's active surveillance protocol. Our inclusion criteria included low risk prostate cancer defined by the following parameters: PSA less than 10, a negative DRE or a localized nodule (T1C/T2A), biopsy Gleason group grade 1 or 2 as defined by the WHO criteria, and unilateral disease involvement on biopsy. Magnetic resonance imaging (MRI) was done to characterize and guide the localization of prostatic lesions for biopsy. Consequently, targeted biopsies were taken from those lesions along with random ones. There was no exclusion criteria based on age. To note, patients that were enrolled into the AS protocol were thoroughly counseled about available treatment options including their possible side effects. Our follow-up strategy based upon a PSA and DRE every 4-6 months, an initial prostate biopsy at the time of enrollment, and a second biopsy at 1 year. Annual MRI was not initially part of the protocol, yet it was introduced in the last few years. Furthermore, an additional re-biopsy was done after the first year with evident signs of clinical progression such as a suspicious rise in PSA, change in DRE, or MRI progression which included the appearance of a new lesion, or an increased

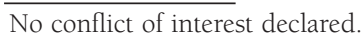


complexity of a previously noted one as defined by the PIRADs system. Descriptive statistics of patients and their above parameters were reported along with a subset analysis of patients that dropped out of the protocol to receive treatment for progression of disease.

\section{Results}

Our populations' age ranged between $51-78$ with a mean of 63.88. PSA values ranged between 1.87-15.9. At presentation, a positive DRE was present in $27.9 \%$ of included patients, whereas negative in $72.1 \%$ of them. Initial MRI's harbored a PIRADS 3 lesions in 14\% of patients, PIRADS 4 in $37.2 \%$, PIRADS 5 in $11.6 \%$. $23.2 \%$ of patients had a negative MRI and $14 \%$ did not have one. The Pathology grade of our patients prostate biopsy revealed a WHO group 1 in $86 \%$ of patients while a WHO group 2 in $14 \%$ of the biopsies (Table 1).

Upon regular follow-up, $46.5 \%$ of patients (20/43) had a significant rise in follow-up PSA, 46.5\% (20/43) had a stable PSA throughout, and 7\% (3/43) did not have a fol-

Table 1.

Mean age, mean PSA, rate of positive DRE, MRI results and pathology grade of our population.

\begin{tabular}{|l|c|}
\hline Patient demographics & Results \\
\hline AGE & $63.88(51-78)$ \\
\hline PSA & $6.26(1.87-15.9)$ \\
\hline DRE (negative) & $72.1 \%(31 / 43)$ \\
\hline DRE (positive) & $27.9 \%(12 / 43)$ \\
\hline MRI (not done) & $14 \%(6 / 43)$ \\
\hline MRI (negative) & $23.2 \%(10 / 43)$ \\
\hline MRI (PIRADS 3) & $14 \%(6 / 43)$ \\
\hline MRI (PIRADS 4) & $37.2 \%(16 / 43)$ \\
\hline MRI (PIRADS 5) & $11.6 \%(5 / 43)$ \\
\hline Pathology grade & \\
\hline WH0 group 1 & $86 \%(37 / 43)$ \\
\hline WH0 group 2 & $14 \%(6 / 43)$ \\
\hline
\end{tabular}

Table 2.

PSA, MRI and biopsies at follow-up.

\begin{tabular}{|l|c|c|c|}
\hline Clinical parameter & Progressed & Stable & N/A \\
\hline PSA & $46.5 \%(20 / 43)$ & $46.5 \%(20 / 43)$ & $7 \%(3 / 43)$ \\
\hline MRI & $18.6 \%(8 / 43)$ & $39.5 \%(17 / 43)$ & $41.9 \%(18 / 43)$ \\
\hline Pathology & $11.6 \%(5 / 43)$ & $46.5 \%(20 / 43)$ & $41.9 \%(18 / 43)$ \\
\hline
\end{tabular}

Table 3.

Comparison of our results to various North American cohorts studied in different medical centers.

\begin{tabular}{|l|c|c|c|c|c|}
\hline Center & $\begin{array}{c}\text { Toronto } \\
(\mathbf{8})\end{array}$ & $\begin{array}{c}\text { Hopkins } \\
(\mathbf{2 9})\end{array}$ & $\begin{array}{c}\text { UCSF } \\
\mathbf{( 1 0 )}\end{array}$ & $\begin{array}{c}\text { Canary pass } \\
\mathbf{( 3 0 )}\end{array}$ & AUBMC \\
\hline No of patients & 993 & 1298 & 321 & 905 & 43 \\
\hline Median age & 68 & 66 & 63 & 63 & 65 \\
\hline Median follow-up & 77 & 60 & 43 & 28 & 40 \\
\hline Overall sunvival & $80 \%$ & $93 \%$ & $98 \%$ & & $\mathrm{~N} / \mathrm{A}$ \\
\hline Cancer specific sunvival & $98 \%$ & $99.90 \%$ & $100 \%$ & & $100 \%$ \\
\hline Conversion to treatment & $36.50 \%$ & $50 \%$ & $24 \%$ & $19 \%$ & $20.90 \%$ \\
\hline (ROT) Gleason grade change & $9.50 \%$ & $15.10 \%$ & $38 \%$ & & $11.60 \%$ \\
\hline (ROT) PSA increase & $11.70 \%$ & & $26 \%$ & & $4.65 \%$ \\
\hline (ROT) Positive LNS & & $0.40 \%$ & & & \\
\hline (ROT) Personal choice & $1.60 \%$ & $8 \%$ & $8 \%$ & & $4.65 \%$ \\
\hline
\end{tabular}

Figure 1.

Mode of intervention after dropping out from active surveillance.

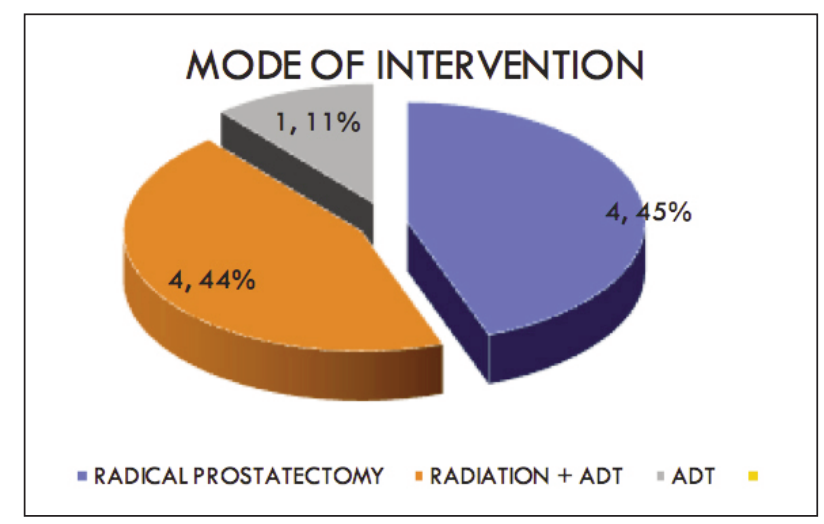

low-up PSA. Out of the prostate MRIs done, 18.6\% (8/43) had imaging progression as per the previously stated criteria, $39.5 \%(17 / 43)$ of lesions were stable, and $41.9 \%$ (18/43) did not have a follow-up MRI. With regards to the follow-up biopsies, $11.6 \%$ (5/43) were upgraded to a higher WHO group, $46.5 \%$ (20/43) were either stable or at a lower group, while $41.9 \%$ (18/43) of the repeat biopsies were not done (Table 2 ).

A descriptive analysis of the patients that dropped out of active surveillance for intervention was performed. 20.9\% (9/43) of patients dropped out. Analysis according to changes in clinical parameters was performed and demonstrated the following: of the 20 patients who progressed by PSA, only 2 patients (10\%) dropped out of active surveillance for intervention. Of the 8 patients that progressed on follow-up MRI, 3 patients (37.5\%) dropped out for intervention. Of the 3 patients who were upgraded on followup biopsies, 2 patients (66.7\%) dropped out for intervention (Figure 1). Two patients elected to drop out and seek intervention due to psychological/patient preference.

Of the patients that dropped out of active surveillance, $44.4 \%$ (4/9) underwent radical prostatectomy, $44.4 \%$ (4/9) underwent radiation therapy plus ADT, and $11.1 \%$ (1/9) took ADT alone (Figure 1). Of those who underwent radical prostatectomy, 50\% (2/4) were upgraded according to the final pathology, while 50\% (2/4) maintained the same grade as the last biopsy result.

\section{Discussion}

According to the NCCN 2019 guidelines, inclusion criteria for active surveillance for prostate cancer includes patients with very low/low-risk disease or intermediate risk disease with low volume disease and a outcome with a life expectancy of 10 years and beyond $(7,8)$. Follow up criteria vary from one institution to another where most opt for a follow-up PSA every 6 months, DRE every 12 months, and biopsy every 1-2 years. Some protocols have incorporated the use of MRI every 12 months or more (NCCN) (9). Our protocol has been consistent with the above recommendations whilst gradually incorporating MRI as a valuable aid in decision making.

Serum marker PSA is the frontline parameter that triggers further workup to rule out clinical progression. This was 
similarly evident in our cohort whereby almost half $(46.5 \%)$ of our patients had a significant rise in PSA that led to the decision of further investigation that included a rebiopsy +/- MRI. Of those with PSA progression, 10\% $(2 / 20)$ had an upgraded pathology on repeat biopsy. One remained eligible for the AS group, while the other was reclassified from low to intermediate risk group leading to an intervention. It is prudent to follow-up biannually with PSA results; however, it is rarely the sole trigger for intervention. When suspicion arises, further clinical workup is warranted via MRI and biopsy $(10,11)$.

With regards to the initial DRE, all patients were either negative $(72.1 \%)$ or suspicious to have a T2a lesion on exam (27.9\%). Patients with large nodules, bilateral nodules, or involvement of seminal vesicles were excluded from active surveillance (12). Abnormal DREs are associated with an increased risk of detecting high-grade disease (12). Any sign of T3 disease or progression in the clinical stage suggested by a DRE would warrant a further investigation/intervention in patients with AS (13). Nevertheless, none of our patients revealed signs of progression upon DRE.

The addition of MRI evaluation proved to be a resourceful tool in clinical decision making for AS patients (14-20).

It was similarly essential for our patients. Upon follow-up, $18.6 \%(8 / 43)$ of our patients progressed on MRI, 37.5\% (3/8) of those patients that progressed were dropped from AS. One of those (1/3), had a suspicious capsule involvement on MRI leading to direct intervention, whereas the other two (2/3) patients had a rebiopsy that revealed upgrading of disease leading to intervention. From the total number of patients, only $13 \%$ of them were not imaged with MRI despite strong recommendation. Imaging for prostate cancer, MRI has been integral for decision making in AS, as it significantly improved detection rates of suspicious lesions. Biopsy without MRI has a misclassification rate of 20-30\% (14). In addition, Berglund et al. showed that an immediate confirmatory biopsy for AS patients revealed an upgrade in 27\% of cases to Gleason 7 and above (15). As such, an MRI is deemed a crucial addition in the diagnostics of prostate cancer as it can accurately guide targeting of clinically significant lesions in $2 / 3$ of men eligible for active surveillance (16). In addition, MRI has a high negative predictive value $90-100 \%$ (17), it also lacks sensitivity to low grade tumors of Gleason $6(3+3)$. Therefore, on initial workup a negative MRI may omit the need for biopsy to rule out prostate cancer and may even be an attractive alternative of recurrent biopsies leading to a decreased incidence of diagnosing low grade prostate cancer $(18,19)$. Yet, the use of MRI for follow-up on AS patients should be subject to better defined radiological parameters (20).

Biopsy results belonging to WHO group 1 or 2 is the final determinant of patient inclusion to the AS protocol of our study. On follow-up, any suspicious clinical parameter would warrant a repeat biopsy. Of those, $11.6 \%(5 / 43)$ were upgraded. Of the upgraded biopsies $80 \%$ (4/5) lead to an intervention. In addition, biopsy result was the trigger for intervention in $44.4 \%$ of patients that dropped out of AS. In a $41.9 \%(20 / 43)$ of follow-up, biopsies at one year were not done. This high percentage can be explained by lack of patient commitment to the AS protocol mainly due to discomfort from undergoing a repeat biopsy as well as false reassurance from the other non- invasive clinical parameters. To note, none of the patients who underwent radical prostatectomy as the intervention of choice had a high-grade pathology specimen (Gleason 8 or above). Biopsy is indeed the most solid parameter for decision making. According to the PRIAS study, switch to active treatment should be guided by biopsy upgrading and/or clinical T3 disease (13). We compared our results to various North American cohorts studied in different medical centers. Despite our small sample size, our numbers were consistent with their results. Fortunately, our OS and CSS revealed no deceased patients. This could be explained by our relatively short median follow-up time of 40 months and our small sample size. Moreover, our conversion to treatment rate was $20.9 \%$ which was comparable to other cohorts (19-50\%) (Table 3).

Truly one of the biggest and main challenges in Prostate cancer is differentiating low risk pathology from aggressive ones. Gleason pathology is currently the most reliable method. MRI has an emerging role in aiding clinicians and is gaining popularity as new studies are in favor of its diagnostic potential. Biomarkers appear to be promising but await prospective studies to be fully endorsed (1). PCA3 and TMPRSS2:ERG may be able to assess risk of aggressive disease yet fail to reveal an independent predictive value or benefit over PSA (21). 4k score has a significant association with reclassification biopsy; however, it showed no additional benefit over PSA in guiding follow-up biopsies in AS (22). Genomic markers include 3 genetic tissue assays that are currently FDA approved. The DECIFER genomic classifier which consists of 22 genes, gives a score $0-1$ and classifies patients into 3 risk groups (23). Genomic Prostate score consists of 17 genes and may potentially aid the initial decision for AS enrollment (24). Cell cycle progression test may similarly aid the decision-making process (25). None have been validated for use in AS; however, they may be incorporated in nomograms especially in deciding on AS for intermediate risk patients (26). Patients with BRCAl/2 mutations are not recommended to undergo active surveillance. When present, these mutations are associated with increased risk of nodal and distant metastasis as well as poor survival outcomes (27).

Our AS protocol has some limitations. Despite an agreed upon consensus on the active surveillance protocol, there was an inter patient variability driven by patient compliance or financial/insurance coverage. In addition, treating physicians in our region are still reluctant to initiate active surveillance protocol for various reasons (28). Moreover, a bigger sample size and longer follow-up period would further solidify our findings and improve our understanding of long-term patient outcomes on active surveillance $(29,30)$.

\section{Conclusions}

In conclusion, active surveillance is a practical and appropriate clinical strategy that should be further employed as part of our patient care arsenal. It is a complex and demanding process for both physician and patient alike as it requires rigorous follow-up with shrewd attention to multiple combined clinical parameters as well as patient commitment and willingness to undergo periodic assessments. PSA is an essential clinical marker for Active Surveillance that may subtly guide decision making. 
MRI is a useful clinical parameter in AS that may obviate need for re-biopsy or even an initial biopsy for prostate cancer. DRE and biopsy are major contributors in halting AS and proceeding towards an intervention especially with evident disease progression either by an upgraded Gleason score or an upstaging upon exam (cT3).

\section{REFERENCES}

1. Briganti A, Fossati N, Catto JWF, et al. Active surveillance for lowrisk prostate cancer: the European Association of Urology position in 2018. Eur Urol. 2018; 74:357-68.

2. Popiolek M, Rider JR, Andren O, et al. Natural history of early, localized prostate cancer: a final report from three decades of follow-up. Eur Urol. 2013; 63:428-35.

3. Albertsen PC, Hanley JA, Fine J. 20-year outcomes following conservative management of clinically localized prostate cancer. Jama. 2005, 293:2095-101

4. Johansson E, Steineck G, Holmberg L, et al. Long-term quality-of-life outcomes after radical prostatectomy or watchful waiting: the Scandinavian Prostate Cancer Group-4 randomised trial. The Lancet Oncology. 2011; 12:891-9.

5. Grossman DC, Curry SJ, Owens DK, et al. Screening for prostate cancer: US preventive services task force recommendation statement. JAMA. 2018; 319:1901-13.

6. Hamdy FC, Donovan JL, Lane JA, et al. 10-year outcomes after monitoring, surgery, or radiotherapy for localized prostate cancer. New Engl J Med 2016; 375:1415-24.

7. Klotz L. Active surveillance in intermediate-risk prostate cancer. BJU Int. 2020; 125:346-54.

8. Klotz L, Vesprini D, Sethukavalan P, et al. Long-term follow-up of a large active surveillance cohort of patients with prostate cancer. J Clin Oncol. 2015; 33:272-7.

9. Carroll PR, Parsons JK, Andriole G, et al. NCCN Guidelines insights: prostate cancer early detection, Version 2.2016. JNCCN. 2016; 14:50919.

10. Welty CJ, Cowan JE, Nguyen H, et al. Extended followup and risk factors for disease reclassification in a large active surveillance cohort for localized prostate cancer. J Urol 2015; 193:807-11.

11. Garisto JD, Klotz L. Active surveillance for prostate cancer: how to do it ight. Oncology (Williston Park) 2017; 31:333-40.

12. Gosselaar C, Roobol MJ, Roemeling S, Schroder FH. The role of the digital rectal examination in subsequent screening visits in the European randomized study of screening for prostate cancer (ERSPC), Rotterdam. Eur Urol. 2008; 54:581-8.

13. Bokhorst LP, Lepisto I, Kakehi Y, et al. Complications after prostate biopsies in men on active surveillance and its effects on receiving further biopsies in the Prostate cancer Research International: Active Surveillance (PRIAS) study. BJU Int. 2016; 118:366-71.

14. Ploussard G, Salomon L, Xylinas E, et al. Pathological findings and prostate specific antigen outcomes after radical prostatectomy in men eligible for active surveillance--does the risk of misclassification vary according to biopsy criteria? J Urol. 2010; 183:539-44.

15. Berglund RK, Masterson TA, Vora KC, et al. Pathological upgrading and up staging with immediate repeat biopsy in patients eligible for active surveillance. J Urol 2008; 180:1964-7.

16. De Rooij M, Hamoen EH, Futterer JJ, et al. Accuracy of multiparametric MRI for prostate cancer detection: a meta-analysis. AJR 2014; 202:343-51.

17. Gaziev G, Wadhwa K, Barrett T, et al. Defining the learning curve for multiparametric magnetic resonance imaging (MRI) of the prostate using MRI-transrectal ultrasonography (TRUS) fusion-guided transperineal prostate biopsies as a validation tool. BJU Int. 2016; 117:80-6.

18. Barentsz JO, Richenberg J, Clements R, et al. ESUR prostate MR guidelines 2012. Eur Radiol. 2012; 22:746-57.

19. Dianat SS, Carter HB, Pienta KJ, et al. Magnetic resonance-invisible versus magnetic resonance-visible prostate cancer in active surveillance: a preliminary report on disease outcomes. Urology. 2015; 85:147-53.

20. Schoots IG, Petrides N, Giganti F, et al. Magnetic resonance imaging in active surveillance of prostate cancer: a systematic review. Eur Urol. 2015; 67:627-36

21. Lin DW, Newcomb LF, Brown EC, et al. Urinary TMPRSS2:ERG and PCA3 in an active surveillance cohort: results from a baseline analysis in the Canary Prostate Active Surveillance Study. Clin Cancer Res 2013; 19:2442-50.

22. Lin DW, Newcomb LF, Brown MD, et al. Evaluating the four kallikrein panel of the 4Kscore for prediction of high-grade prostate cancer in men in the Canary Prostate Active Surveillance Study. Eur Urol. 2017; $72: 448-54$

23. Klein EA, Haddad Z, Yousefi K, et al. Decipher genomic classifier measured on prostate biopsy predicts metastasis risk. Urology. 2016; 90:148-52.

24. Klein EA, Cooperberg MR, Magi-Galluzzi C, et al. A 17-gene assay to predict prostate cancer aggressiveness in the context of Gleason grade heterogeneity, tumor multifocality, and biopsy undersampling. Eur Urol. $2014 ; 66: 550-60$

25. Shore ND, Kella N, Moran B, et al. Impact of the cell cycle progression test on physician and patient treatment selection for localized prostate cancer. J Urol. 2016; 195:612-8.

26. Wang SY, Cowan JE, Cary KC, et al. Limited ability of existing nomograms to predict outcomes in men undergoing active surveillance for prostate cancer. BJU Int. 2014; 114:E18-e24.

27. Castro E, Goh C, Olmos D, et al. Germline BRCA mutations are associated with higher risk of nodal involvement, distant metastasis, and poor survival outcomes in prostate cancer. J Clin Oncol. 2013; 31:1748-57

28. El Sebaaly R, Mansour M, Labban M, et al. Survey on the practice of active surveillance for prostate cancer from the Middle East. Prostate Int. 2020; 8:41-8.

29. Tosoian JJ, Mamawala M, Epstein JI, et al. Intermediate and longerterm outcomes from a prospective active-surveillance program for favorable-risk prostate cancer. J Clin Oncol. 2015; 33:3379-85.

30. Newcomb LF, Thompson IM, Jr., Boyer HD, et al. Outcomes of active surveillance for clinically localized prostate cancer in the prospective, Multi-Institutional Canary PASS Cohort. J Urol. 2016; $195: 313-20$

\section{Correspondence}

Mohammad Hout, MD - mh21@aub.edu.lb

Ali Merhe, MD - am134@aub.edu.lb

Nassib Fares Heidar, MD - nal92@aub.edu.lb

Jose M El-Asmar, MD - je56@aub.edu.lb

Wassim Wazzan, MD - ww04@aub.edu.lb

Bassel Bachir,MD - bb12@aub.edu.lb

AlbertEl-Haji, MD - ae67@aub.edu.lb

Muhammad Bulbul, MD, Clinical Professor (Corresponding Author) mb30@aub.edu.lb

Department of Urology, American University of Beirut Medical Center Riad El-Solh 11072020 Beirut, Lebanon

Rola Jaafar, MD - rj29@aub.edu.lb

Department of Surgery, American University of Beirut Medical Center, Beirut, Lebanon 